\title{
Deterministic Relativistic Quantum Bit Commitment
}

\author{
Emily Adlam ${ }^{1}$ and Adrian Kent ${ }^{1,2}$ \\ ${ }^{1}$ Centre for Quantum Information and Foundations, DAMTP, Centre for Mathematical Sciences, \\ University of Cambridge, Wilberforce Road, Cambridge, CB3 0WA, U.K. \\ ${ }^{2}$ Perimeter Institute for Theoretical Physics, 31 Caroline Street North, Waterloo, ON N2L 2Y5, Canada.
}

(Dated: April 2015)

\begin{abstract}
We describe new unconditionally secure bit commitment schemes whose security is based on Minkowski causality and the monogamy of quantum entanglement. We first describe an ideal scheme that is purely deterministic, in the sense that neither party needs to generate any secret randomness at any stage. We also describe a variant that allows the committer to proceed deterministically, requires only local randomness generation from the receiver, and allows the commitment to be verified in the neighbourhood of the unveiling point. We show that these schemes still offer nearperfect security in the presence of losses and errors, which can be made perfect if the committer uses an extra single random secret bit. We discuss scenarios where these advantages are significant.
\end{abstract}

Introduction Relativistic quantum cryptography exploits the combined power of Minkowski causality and quantum information theory to control information in order to implement cryptographic tasks. A variety of interesting tasks (e.g. [13 19]) are now known to be achievable, either with unconditional security or with security significantly enhanced relative to classical protocols. There has also been progress in characterising fundamental constraints imposed on quantum information tasks by Minkowski causality [10 12].

The first significant application of relativistic cryptography was to bit commitment [8, 9, 13, 28], a basic cryptographic primitive which has many applications and which cannot be implemented securely by using quantum information alone 2 27]. Several classical and quantum relativistic bit commitment protocols have now been proven secure [9, 11, 13, 20 22, 28]. The feasibility of secure relativistic quantum bit commitment has also been demonstrated experimentally [22, 23]. The feasibility of classical relativistic bit commitment has also been investigated [9, 24] with a view to near term implementation [24].

Nonetheless, the full range of possibilities for relativistic quantum bit commitment protocols has not yet been systematically explored, nor are all the possible tradeoffs between security advantages and requirements well understood. We are motivated to address these questions both because they are practically relevant and because the answers illuminate the general properties of relativistic quantum information and its relationship to cryptography.

Existing relativistic classical and quantum bit commitment protocols [9, 13, 28] require at least one party to locally generate and then securely store and/or distribute secret classical random strings. While this is a reasonable capability to assume in many cryptographic contexts, it may not always be practical. For example, if protocols are being implemented over a network of many sites, it may not necessarily be desirable to set up random number generators or secure classical memories at every site.

One might at first think that quantum protocols cannot have any advantage here, since if a party can securely and reliably prepare, distribute and measure entangled quantum states, they can obtain secure classical random strings from those states as and when required. In many scenarios this argument may indeed apply. However, quantum information has security advantages compared to classical information, particularly when one considers a protocol as part of a larger cryptographic exchange. For example, if a party is concerned that there has been a security breach at one of their sites, they can check whether a distributed quantum state remains in the correct form, whereas they cannot tell for sure whether a purportedly secret distributed classical random string has been read at some location by an adversary.

These points, alongside interest in understanding better theoretically the relationship between relativistic quantum information and cryptography, motivate us to consider relativistic quantum bit commitment protocols that require less secret classical randomness, or even none. We describe here two entanglement-based relativistic bit commitment protocols that minimize the need for classical randomness: indeed, one of them, in its ideal form, requires no randomness at all. Their security can be understood as a consequence of the monogamy of quantum entanglement.

Bit commitment A bit commitment protocol involves two mistrustful parties who control disjoint secure regions (laboratories) and exchange information. The committer, Alice, carries out actions that commit her to a particular bit value (or, in the quantum case, a particular superposition of bit values). She can later, if she chooses, give the receiver, Bob, classical or quantum information that unveils the committed bit. Ideally, the protocol should rely only on physical principles to guarantee to Bob that Alice is committed by her initial actions, and to Alice that Bob can learn no information about the committed bit unless and until she unveils.

When considering relativistic bit commitment protocols, these definitions need to be framed more carefully [11]. In such protocols, both Alice and Bob are represented by networks of collaborating agents distributed appropriately in space-time. All of Alice's agents are assumed to be acting with perfect trust in one another. However, at any given 
time (in some fixed reference frame), they do not necessarily all have the same information, both because they are separated in space and because quantum information cannot be broadcast. The same applies to Bob's agents.

In standard relativistic bit commitment protocols, the commitment is carried out by one of Alice's agents. In an idealized model, this agent acts at a single point in space-time; more realistically she acts within a spatially small secure laboratory during a small time interval. The unveiling may be carried out by any number of Alice's agents, possibly including the committing agent. In principle a protocol could require agents to follow any specified causal paths in space-time. However, we usually assume there is a natural inertial frame with respect to which they are all stationary, so that they are located at fixed points in space (or within fixed small laboratories) throughout the protocol. Since we allow arbitrary numbers of agents, this loses no generality, so long as we assume that Alice's agents have secure classical and quantum communication channels. (Note, however, that this last assumption may not always be justified; if not, the possibility of mobile agents should be kept in mind.)

Security definitions One needs to be careful about what, precisely, a bit commitment protocol is intended to guarantee in relativistic scenarios. Specifically, one needs to be clear which agent (or combination of agents) is (are) committed at which point(s). We follow the physically motivated definition first set out in Ref. [11], which requires that a bit commitment should guarantee that the committed data was available to and input by Alice's committing agent $A_{c}$ at the space-time point where the commitment occurs. This definition allows for the possibility of $A_{c}$ inputting a quantum superposition of the values 0 and 1 . However, it excludes protocols in which the unveiling agents could influence the value of the unveiled bit by using correlated information that they acquired independently of $A_{0}[1] .{ }^{1}$

Let the agents involved in the unveiling be $A_{i}(i=0,1, \ldots)$. Let $p_{0}(S)$ and $p_{1}(S)$ be the probabilities that, by following some collective strategy $S$, they persuade Bob that, according to the rules of the protocol, they have validly unveiled 0 or 1 respectively.

We say a relativistic quantum bit commitment protocol is unconditionally secure against Alice if, given any commitment actions by $A_{c}$ that Bob will accept as valid, and any strategies $S$ and $S^{\prime}$ by the unveiling agents $A_{i}$ that are allowed by quantum theory and special relativity, we have $p_{0}(S)+p_{1}\left(S^{\prime}\right)<1+\epsilon(N)$, where $N$ is a variable security parameter of the protocol and $\epsilon(N) \rightarrow 0$ as $N \rightarrow \infty$.

In the protocols we consider below, there are two unveiling agents $A_{0}$ and $A_{1}$, whose actions are spacelike separated from each other and from those of $A_{c}$. The probability of a successful unveiling of bit value $i$ depends only on the actions of agent $A_{i}$. A collective strategy $S$ may be fixed by Alice before the protocol, or Alice's agents responsible for unveiling 0 and 1 may independently choose their strategies after the commitment time, possibly conditioned on events in the past lightcone of their verification point but not of the commitment point. We subsume the latter possibility under the former by allowing any strategy $S$ to include steps in which agents make strategic choices with probabilities conditional on certain external events, with those events themselves now explicitly included in the description of strategy $S$. Any strategy whereupon the conditional probabilities for these choices are nontrivial may be written as a convex combination of deterministic strategies, so no probabilistic strategy can have greater success probability than the most successful deterministic strategy.

For protocols of the type we consider we can thus simplify the above definition: such a protocol provides unconditional security against Alice if any only if for any collective strategy $S$ which is possible according to quantum theory and special relativity, $p_{0}(S)+p_{1}(S)<1+\epsilon(N)$ and $\epsilon(N) \rightarrow 0$ as $N \rightarrow \infty$, where $N$ is a variable security parameter of the protocol, and $p_{0}(S)$ and $p_{1}(S)$ are the probabilities that, by following strategy $S$, Alice and her agents persuade Bob that they have validly unveiled 0 or 1 respectively according to the rules of the protocol.

We say a relativistic bit commitment protocol is unconditionally secure against Bob if, whatever strategy Bob's agents follow, if Alice's agents choose not to unveil, then the probability of any of Bob's agents correctly guessing the committed bit at any point in space-time is bounded by $1 / 2+\epsilon^{\prime}(N)$, where $\epsilon^{\prime}(N) \rightarrow 0$ as $N \rightarrow \infty$. It follows from this definition, by the no-signalling principle, that when Alice does choose to unveil, Bob cannot guess Alices commitment anywhere that does not lie in the future lightcone of the unveiling points.

In the protocols we consider below, Alice has one committing agent, $A_{c}$, and two unveiling agents, $A_{0}$ and $A_{1}$, who can unveil a valid commitment to $b=0$ and 1 respectively. An additional security criterion may be required for such protocols: that if $A_{c}$ does not make a valid commitment to bit value $b, A_{b}$ follows the unveiling protocol and $A_{\bar{b}}$ does not, then Bob's agents, at any point in space-time, should gain no information about whether $A_{c}$ committed to bit value $\bar{b}$ or declined to make a valid commitment. As we explain below, with simple modifications, our protocols also satisfy this criterion.

Relation of commitment and unveiling points Another issue is what exactly is meant by the unveiling taking place "later" than the commitment in Minkowski space. In some quantum relativistic bit commitment protocols

\footnotetext{
${ }^{1}$ Following Ref. [1], another discussion of security definitions from a somewhat different perspective was given in Ref. 21].
} 
[13, 28], the unveiling points are in the lightlike causal future of the commitment point. In the idealized case in which agents are pointlike and their actions are instantaneous, these protocols guarantee that the committing agent was committed at the commitment point, in the sense given above. In such protocols, the statement that the unveilings are later than the commitment is true independent of the frame. We call these lightlike causal (LC) relativistic bit commitments.

We wish here also to consider protocols in which the unveiling points are space-like separated from the commitment point. The most obviously interesting case is that in which all unveiling points are later than the commitment point with respect to some fixed frame $F$. We call such protocols fixed frame positive duration (FFPD) relativistic bit commitments.

Generally, if there is a fixed frame $F^{\prime}$ in which all the agents are stationary during the protocol, we will take $F^{\prime}=F$. One motivation for considering this case is that it allows us to consider sequences of protocols in which the unveiling points tend towards the future light cone, and so to relate LC and FFPD commitments. Another is that there are many practical situations - such as protocols carried out on terrestrial computer networks - in which there is a generally agreed (approximately) inertial frame and time coordinate. In such scenarios, commitments are potentially useful provided they have a positive duration with respect to this coordinate. A third motivation is the possibility of sustaining a bit commitment for several rounds by using sequences of protocols with space-like separations, as in the examples of Refs. [8, 9]. In this case, the geometry can be chosen so that any or all possible final unveiling points are in the causal future of the commitment point. A sequence of LC and/or FFPD relativistic bit commitments can thus produce a timelike causal (TC) relativistic bit commitment: that is, a commitment in which all the unveiling points are in the timelike future of the commitment point.

As usual in quantum cryptography, we initially present our protocols in an idealized form assuming perfect quantum state preparations, transmissions, measurements and computations. However, the protocols are tolerant to errors and losses, as we discuss later.

Space-time and communications We also make standard idealizations about the background geometry and signalling speed. We suppose that space-time is Minkowski and that Alice and Bob each have agents in secure laboratories infinitesimally separated from the points $P, Q_{0}$ and $Q_{1}$, that signals are sent at precisely light speed, and that all information processing is instantaneous. Again, these assumptions can be relaxed. The protocols remain secure in realistic implementations with finite separations and near light speed communication. If these corrections are small, the only significant effect is that Bob is guaranteed that Alice's commitment is binding from some point $P^{\prime}$ in the near causal future of $P$, rather than from $P$ itself [13]. Allowing for small deviations from Minkowski geometry also requires small corrections to the geometry when stating the security guarantees, but does not essentially affect security beyond that $[9]$.

Geometry Alice and Bob agree on a space-time point $P$, an inertial set of coordinates $(x, y, z, t)$ for Minkowski space, with $P$ as the origin. We focus here on the simplest case in which there are two possible unveiling points $Q_{0}$ and $Q_{1}$, both space-like separated from $P$ : the protocols straightforwardly extend to versions with $N$ unveiling points committing $\log (N)$ bits. Alice and Bob each have agents, who during the protocol are separated in secure laboratories, adjacent to each of the points $P, Q_{0}, Q_{1}$. To simplify for the moment, we take the distances from these labs to the relevant points as negligible. Although it is not necessary for much of our discussion, we assume that $Q_{0}$ and $Q_{1}$ have positive time coordinates in the given frame, so as to define FFPD relativistic bit commitments. Let the agents adjacent to $P$ be $A_{c}$ and $B_{c}$, and those adjacent to $Q_{i}$ be $A_{i}$ and $B_{i}$.

In the following protocols, for definiteness, we describe a procedure in which Alice and her agents exchange qubits by secure physical transportation in the preparation phase. However, they may alternatively employ teleportation or a secure quantum channel without significantly altering the protocols' security. Likewise Bob and his agents may exchange qubits by any secure means. Bob may also arrange to combine his qubits at a variety of locations, depending on where he wishes to verify the unveiled bit.

\section{ETBC: Simple Entanglement transfer protocol}

Preparation 1. $\quad A_{c}$ prepares a total of $2 N$ Bell pairs in the state $\Psi^{-}$; let the qubits in the first $N$ pairs be $\left(W_{0 P}^{j}, W_{0 Q}^{j}\right)$ and the second $N$ pairs $\left(W_{1 P}^{j}, W_{1 Q}^{j}\right)$, where $j \in[1, N]$. She retains the qubits $W_{i P}^{j}$, gives the qubits $W_{0 Q}^{j}$ to $A_{0}$ and gives the qubits $W_{1 Q}^{j}$ to $A_{1}$.

2. $A_{0}$ and $A_{1}$ travel to locations adjacent to the spatial coordinates of $Q_{0}$ and $Q_{1}$. We assume that $A_{c}, A_{0}$ and $A_{1}$ have secure laboratories that protect their qubits, so Bob cannot interfere with them in any way after the initial preparation. In particular, $A_{0}$ and $A_{1}$ travel within secure laboratories.

Commitment At the designated commitment point $P, A_{c}$ gives $B_{c}$ a set of $N$ labelled qubits $Q_{a}^{j}$. If she wishes to commit to bit value $i$, these are the qubits $W_{i P}^{j}$, for $j \in[1, N]$, labelled in sequence.

Unveiling If the agent $A_{i}$ believes Alice wishes to unveil, she gives the labelled qubits $W_{i Q}^{j}$ to Bob's agent $B_{i}$. $A_{c}$ (and/or, if preferred, one or both of the $A_{i}$ ) also sends to Bob's neighbouring agent a classical message stating the 
bit value $b$. (Note that in principle the agents $A_{c}, A_{0}$ and $A_{1}$ may make these decisions independently. To coordinate them and ensure that all or none unveil, Alice needs to give them instructions in advance. These instructions could depend on separate events in the past light cones of their unveiling decision points, if Alice knows these events will be correlated.)

Verification Once at least one of Bob's agents knows the claimed bit value $b$, they securely transmit to one agent (for example $B_{c}$ or $B_{b}$ ) all the qubits given to $B_{c}$ and to $B_{b}$. The receiving agent then carries out projective measurements in the Bell basis on the qubits $\left(Q_{a}^{j}, W_{b Q}^{j}\right)$ for each $j \in[1, N]$. If they get outcomes corresponding to the Bell state $\Psi^{-}$for all $j$, Bob accepts that Alice made a valid commitment to bit value $b$. (As noted above, this verification step can be carried out at a location of Bob's choice: for example, it could be made by an agent half-way between $B_{c}$ and $B_{b}$.)

Security against Alice We prove security against Alice assuming the validity of quantum mechanics and assuming that Bob's measuring devices are reliable. (Neither this protocol nor the variation considered below gives Bob device independent security or security against adversaries who can exploit hypothetical post-quantum non-signalling theories.)

Write the Hilbert spaces for the $N$ qubits held by $B_{0}, B_{1}$ and $B_{c}$ as $H_{0}, H_{1}$ and $H_{2}$ respectively, and write $H=H_{1} \otimes H_{2} \otimes H_{0}$. Bob tests for a purported commitment to zero by a measurement defined by the projection

$$
P_{0}=\otimes_{j=1}^{N}\left(I_{1}^{j} \otimes\left|\Psi_{-}\right\rangle_{20}^{j}\left\langle\left.\Psi_{-}\right|_{20} ^{j}\right) .\right.
$$

Bob tests for a purported commitment to one by a measurement defined by the projection

$$
P_{1}=\otimes_{j=1}^{N}\left(\left|\Psi_{-}\right\rangle_{12}^{j}\left\langle\left.\Psi_{-}\right|_{12} ^{j} \otimes I_{0}^{j}\right) .\right.
$$

Here $I_{k}^{j}$ is the identity operator on the $j$-th qubit in $H_{k}$ and $\left|\Psi_{-}\right\rangle_{k l}^{j}$ is a Bell state of the $j$-th qubits in $H_{k} \otimes H_{l}$. The operator $Q=P_{0} P_{1}$ can be written as $Q=\otimes_{j=1}^{N} Q_{j}$, where $Q_{j}$ acts on the triple of $j$-th qubits from each Hilbert space and has operator norm $\left|Q_{j}\right|=1 / 2$; hence $Q$ has operator norm $|Q|=2^{-N}$.

For any state $|\psi\rangle$ defining triples of $N$ qubits that Alice might hand over to $B_{c}, B_{0}$ and $B_{1}$, we thus have

$$
\begin{aligned}
|Q| \psi\rangle \mid & \left.=\left|P_{0}\right| \psi\right\rangle-P_{0}\left(1-P_{1}\right)|\psi\rangle \mid \\
& \left.\geq\left|P_{0}\right| \psi\right\rangle|-| P_{0}\left(1-P_{1}\right)|\psi\rangle \mid \\
& \left.\geq\left|P_{0}\right| \psi\right\rangle|-|\left(1-P_{1}\right)|\psi\rangle \mid \\
& \left.\geq\left(p_{0}^{1 / 2}-\left(1-p_{1}\right)^{1 / 2}\right)\right)
\end{aligned}
$$

where $p_{0}$ and $p_{1}$ are the respective probabilities of successfully persuading Bob that 0 and 1 was unveiled using the state $|\psi\rangle$.

This gives that $p_{0}+p_{1} \leq 1+2^{-N+1}+2^{-2 N}$. As this holds for any possible state $|\psi\rangle$, it implies security (in the standard sense [9, 13, 28] for a relativistic quantum bit commitment) with security parameter $N$.

Security against Bob At commitment, Bob receives a set of $N$ qubits entangled with another $N$ qubits not in his possession. They have the same reduced state (a uniform mixture) regardless of the committed bit. He thus cannot obtain any information about the bit before unveiling.

ETRBC: Entanglement transfer protocol with randomisation In this variation, Alice follows the protocol above, but now $B_{c}$ randomly selects half the qubits given to him to send securely to $B_{0}$, sending the other half to $B_{1}$. This allows both $B_{0}$ and $B_{1}$ to directly test the bit value as soon as they receive these qubits.

Preparation 1. $A_{c}$ prepares $2 N$ Bell pairs, $\left(W_{0 P}^{j}, W_{0 Q}^{j}\right)$ and $\left(W_{1 P}^{j}, W_{1 Q}^{j}\right)$ with $j \in[1, N]$, in the state $\Psi^{-}$. She gives the qubits $W_{0 Q}^{j}$ to $A_{0}$ and the qubits $W_{1 Q}^{j}$ to $A_{1}$. We take $N$ even for simplicity. (The protocol can easily be varied to also allow for odd $N$.)

2. $A_{0}$ and $A_{1}$ travel to locations adjacent to the spatial coordinates of $Q_{0}$ and $Q_{1}$. We assume that $A_{c}, A_{0}$ and $A_{1}$ have secure laboratories that protect their qubits, so Bob cannot interfere with them in any way after the initial preparation. In particular, $A_{0}$ and $A_{1}$ travel within secure laboratories.

Commitment At the designated commitment point $P, A_{c}$ gives $B_{c}$ a set of $\mathrm{N}$ labelled qubits $Q_{a}^{j}$. In order to commit to bit value 0 , she gives him the qubits $W_{0 P}^{j}$; in order to commit to bit value 1 , she gives him the qubits $W_{1 P}^{j}$.

Distribution $B_{c}$ sends a randomly selected size $N / 2$ subset $J_{0}$ of his received qubits to $B_{0}$ and the remaining subset, $J_{1}$, to $B_{1}$. All qubits are sent with the corresponding labels $j$.

Unveiling If the agent $A_{i}$ believes Alice wishes to unveil, she gives the labelled qubits $W_{i Q}^{j}$ to Bob's agent $B_{i}$. $\left(A_{c}\right.$ and/or either or both of the $A_{i}$ may also send to Bob's neighbouring agent a classical message stating the bit value $b$ if they wish, although it is not necessary in this protocol. In any case, as in the previous protocol, some advance instructions from Alice are needed to ensure any unveiling decisions are coordinated.) 
Verification Once he has received the qubits sent by $B_{c}, B_{i}$ carries out projective measurements in the Bell basis on the qubits $\left(Q_{a}^{j}, W_{i Q}^{j}\right)$ for each $j \in J_{i}$. If $B_{i}$ gets outcomes corresponding to the Bell state $\Psi^{-}$for all $j \in J_{i}$ he accepts that Alice made a valid commitment to bit value $i$.

Security against Alice Again, we prove security against Alice assuming the validity of quantum mechanics and assuming that Bob's measuring devices are reliable.

Write the Hilbert spaces for the $N$ qubits held by $B_{0}, B_{1}$ and $B_{c}$ as $H_{0}, H_{1}$ and $H_{2}$ respectively, and write $H=H_{1} \otimes H_{2} \otimes H_{0} . B_{0}$ tests for a commitment of zero by a measurement defined by the projection

$$
P_{0}^{J_{0}}=\otimes_{j \in J_{0}}\left(I_{1}^{j} \otimes\left|\Psi_{-}\right\rangle_{20}^{j}\left\langle\left.\Psi_{-}\right|_{20} ^{j}\right) .\right.
$$

$B_{1}$ tests for a commitment of one by a measurement defined by the projection

$$
P_{1}^{J_{1}}=\otimes_{j \in J_{1}}\left(\left|\Psi_{-}\right\rangle_{12}^{j}\left\langle\left.\Psi_{-}\right|_{12} ^{j} \otimes I_{0}^{j}\right) .\right.
$$

Suppose that Alice prepares a state $|\psi\rangle$ such that the probability of passing the test for zero is $p \geq p_{0}$. Then there must be at least one subset $J_{0}$ for which this probability is at least $p_{0}$, i.e. for which

$$
p_{0}^{J_{0}}=\left\langle\psi\left|P_{0}^{J_{0}}\right| \psi\right\rangle \geq p_{0} .
$$

Consider any subset $J_{0}^{\prime}$ such that $J_{0} \cap J_{0}^{\prime} \leq N / 3$.

By a similar argument to that above, we obtain

$$
\left|P_{0}^{J_{0}} P_{1}^{J_{1}^{\prime}}\right| \leq 2^{-N / 6}
$$

and

$$
p_{1}^{J_{1}^{\prime}} \leq 1+2^{-N / 6+1}+2^{-N / 3}-p_{0}^{J_{0}} \leq 1-p_{0}+2^{-N / 6+1}+2^{-N / 3} .
$$

Now the proportion of subsets $J_{0}^{\prime}$ with $J_{0} \cap J_{0}^{\prime}>N / 3$ falls off exponentially with $N$ : to leading order it is bounded by $(N / 6)\left(2^{-10 / 6} 3\right)^{N}$. Hence the overall probability of bit value one being accepted, $p_{1}$, is bounded by $p_{1} \leq 1-p_{0}+2^{-N / 6+1}+2^{-N / 3}+O\left(N / 6\left(2^{-10 / 6} 3\right)^{N}\right)$, again giving security with security parameter $N$.

Security against Bob As before, at commitment, Bob receives a set of $N$ qubits entangled with another $N$ qubits not in his possession. They have the same reduced state (a uniform mixture) regardless of the committed bit. He thus cannot obtain any information about the bit before unveiling.

Errors and Losses In any realistic implementation, Alice's state preparation and Bob's measurements will be imperfect and their communication channels and storage devices will have some noise and losses. To show that the protocols will be feasible with sufficiently good, but imperfect, technology we need versions adapted to allow for some non-zero level of errors and losses.

We first assume that Bob follows the protocol and measures each purported singlet separately, and that the errors and losses for each singlet are small and statistically independent.

For protocol ETBC, in this error model, Bob can test for a purported commitment of zero, with negligible probability of getting a false negative result, by checking that he gets positive answers for a proportion $(1-\epsilon) N$ of tests for the singlet $\left|\Psi_{-}\right\rangle_{20}$, where $\epsilon>0$ is small. The error model implies that the probability of a state $|\psi\rangle$ passing the test is no more than $\left.\left|P_{0}^{\delta}\right| \psi\right\rangle\left.\right|^{2}+\gamma(\delta, N)$.

Here $P_{0}^{\delta}=\sum_{m=(1-\delta) N}^{N} P_{m}^{0}$, where $\delta>\epsilon$ is also small, and chosen so that $\gamma(\delta, N) \rightarrow 0$ as $N \rightarrow \infty$. The operator $P_{m}^{0}$ is the projection onto the subspace of states spanned by states of the form $\otimes_{i=1}^{N}\left|\Psi_{i}\right\rangle_{20}\left|\Phi_{i}\right\rangle_{1}$, where the $\left|\Psi_{i}\right\rangle_{20}$ are Bell states, of which precisely $m$ are $\left|\Psi_{-}\right\rangle$, and the $\left|\Phi_{i}\right\rangle_{1}$ are arbitrary qubits in $H_{1}$.

Bob similarly tests for a purported commitment of one by checking that he gets positive answers for a proportion $(1-\epsilon) N$ of tests for the singlet $\left|\Psi_{-}\right\rangle_{12}$. The probability of a state $|\psi\rangle$ passing this test is (up to negligible quantities) no more than $\left.\left|P_{1}^{\delta}\right| \psi\right\rangle\left.\right|^{2}+\gamma(\delta, N)$, where $P_{1}^{\delta}=\sum_{m=(1-\delta) N}^{N} P_{m}^{1}$ is defined similarly.

The operator $P_{0}^{\delta}$ can be written as a sum of $\sum_{x=0}^{N \delta} C_{N-x}^{N} 3^{x}$ terms involving one-dimensional projectors onto tensor products of Bell states in $H_{2} \otimes H_{0}$, tensored with the identity on $H_{1}$. The operator $P_{1}^{\delta}$ can be written similarly, using Bell state projections on $H_{0} \otimes H_{1}$. The operator $Q^{\delta}=P_{0}^{\delta} P_{1}^{\delta}$ can thus be written as a sum of $\left(\sum_{x=0}^{N \delta} C_{N-x}^{N} 3^{x}\right)^{2}$ rank one operators, each of which has operator norm no more than $2^{-N+2 \delta N}$. This gives the (weak, but adequate for our purpose) bound $\left|Q^{\delta}\right| \leq 2^{-N+2 \delta N} 3^{2 \delta N}(N \delta+1)^{2}\left(C_{N-N \delta}^{N}\right)^{2}$, which tends to zero for large $N$ and fixed small $\delta$. The security argument then runs as before.

The security proof for protocol ETRBC similarly extends to cover small levels of errors and losses under the assumptions above. 
For completeness, we should note another possible security issue. If the errors in Alice's singlet state preparations vary over time in some predictable way, then the reduced density matrices for the states handed over to $B_{c}$ by $A_{c}$ may also vary predictably. Given a deterministic protocol, we have to assume that the order in which $A_{c}$ labels the singlets after producing them is public information. $B_{c}$ might then be able to infer some information about the committed bit by measuring these states, without waiting to combine them with states returned by the $A_{i}$.

This may not seem a significant practical worry, since in practice one might reasonably expect the predictable component of any variation in Alice's preparation devices to be very small. Moreover, some deterministic strategies could reduce it further. For example, the information revealed by a monotonic drift of some parameter over time could be greatly reduced by taking the odd time ordered singlets produced (the 1st, $3 \mathrm{rd}$, and so on) to be the first $N$ for the protocol, and the even ordered to be the second $N$. Still, any predictable variation prevents perfect security against Bob, according to our definition. This concern can be eliminated if $A_{c}$ groups the states into two batches of $N$ singlets by some deterministic method, and then decides randomly which batch is labelled from 1 to $N$ and which from $N$ to $2 N$. This requires her to generate and keep secure a single random bit.

\section{Discussion}

Ideal case: no losses or errors The first protocol has a theoretically interesting advantage over any previous relativistic bit commitment protocol in that it is deterministic: neither party needs to make any random choices of classical data or quantum states. It thus satisfies the strongest possible form of Kerckhoff's cryptographic principle that a cryptographic system should be secure even if everything about it except the choice of key is public knowledge: here, neither party even needs a secure key. Generating secure randomness is itself a cryptographic problem that requires extra security assumptions, or trusted secure quantum devices, or both. Eliminating any need for it requires fewer resources and removes some potential security issues.

These advantages come at a price. Bob does not know whether Alice will choose to unveil a commitment to 0 or to 1 , and the no-summoning theorem [10] prevents him from having the qubit $Q_{a}$ available at spacelike separated points along the different directions associated with 0 and 1, the time between Alice's unveiling and the earliest time at which Bob can verify her commitment is twice as long for this variation. In time-sensitive situations this may be a disadvantage.

This is what motivates the second version of our protocol. It eliminates this potential drawback by allowing each $B_{i}$ to test whether the bit is $i$ at the earliest possible point, as soon as a light signal from $B_{c}$ reaches them. After these points, Alice has essentially zero probability of both persuading $B_{0}$ that the bit might be 0 and $B_{1}$ that the bit might be 1 . The cost of this advantage is that $B_{c}$ needs to be able to generate a classical random string that is secure, at least in the sense that Alice cannot predict it in advance. The string may be generated immediately after $B_{c}$ receives his qubits from $A_{c}$, and it does not matter if Alice immediately learns the string. This is still less demanding than requiring Bob to generate a secure random quantum state or sequence of states and keep its classical description secure [13, 28]. The protocol also has an advantage over purely classical relativistic protocols [9] in that Alice does not need to generate any secure random data.

Losses and errors As shown, our protocols can be modified to tolerate small losses and errors. The comments above continue to apply, with one small but important qualification. If Alice wishes to eliminate any information leaking to Bob because of potentially predictable variation in Alice's state preparation, our strategy needs $A_{c}$ to generate and keep secure a single random bit for each committed bit. This is a minimal additional security requirement, and needed only to eliminate for what in practice might often be a negligible leakage of information. Still, it should be kept in mind when making comparisons.

Need for trusted devices Both protocols require Bob to rely on his devices to correctly implement projective measurements for Bell states, up to known small levels of losses and errors. The protocols as stated are thus not fully device independent. It also follows that they rely for their security on the validity of quantum theory (not just on the no-signalling principle). However, the protocols can be modified to give device independent versions by replacing verification steps by (for example) CHSH tests: we will give a detailed discussion elsewhere [25].

Other comments Note that, like all technologically unconstrained quantum bit commitment protocols [26, 27], our protocols do not prevent Alice from committing to a quantum superposition of bits. She can simply input a superposition $\alpha|0\rangle+\beta|1\rangle$ into a quantum computer programmed to implement the two relevant quantum measurement interactions for inputs $|0\rangle$ and $|1\rangle$ and to send two copies of the quantum outcome data towards $Q_{0}$ and $Q_{1}$, and keep all the data at the quantum level until (if) she chooses to unveil. This gives her no advantage in stand-alone applications of bit commitment, for example for making a secret prediction: it does, however, mean that one cannot assume that in a task involving bit commitment subprotocols, any unopened bit commitments necessarily had definite classical bit values, even if all unveiled bit commitments produced valid classical unveilings.

As with the protocols of Refs. [9, 13, 28], the present protocols can be chained together in sequence, allowing longer term bit commitments and flexibility in the relation between the commitment and unveiling sites (in particular, they need not be lightlike separated). Full security and efficiency analyses for these chained protocols remain tasks for 
future work.

\section{Acknowledgments}

This work was partially supported by an FQXi mini-grant and by Perimeter Institute for Theoretical Physics. Research at Perimeter Institute is supported by the Government of Canada through Industry Canada and by the Province of Ontario through the Ministry of Research and Innovation.

[1] A. Broadbent and A. Tapp, Information-Theoretically Secure Voting Without an Honest Majority, arXiv:0806.1931.

[2] D. Mayers, Unconditionally secure quantum bit commitment is impossible, Phys. Rev. Lett. 78 $3414-3417$ (1997).

[3] D. Mayers, Unconditionally secure quantum bit commitment is impossible, Proceedings of the Fourth Workshop on Physics and Computation (New England Complex System Inst., Boston, 1996), p. 226.

[4] H.-K. Lo and H. Chau, Is quantum bit commitment really possible?, Phys. Rev. Lett. 78 3410-3413 (1997).

[5] H.-K. Lo and H. Chau, Why quantum bit commitment and ideal quantum coin tossing are impossible, Proceedings of the Fourth Workshop on Physics and Computation (New England Complex System Inst., Boston, 1996), p. 76.

[6] D. Mayers, A. Kitaev and J. Preskill, Superselection rules and quantum protocols, Phys. Rev. A 69052326 (2004).

[7] G. D'Ariano, D. Kretschmann, D. Schlingemann, R. Werner, Reexamination of Quantum Bit Commitment: the Possible and the Impossible, Phys. Rev. A 76, 032328 (2007).

[8] A. Kent, Unconditionally secure bit commitment, Phys. Rev. Lett. 83 1447-1450 (1999).

[9] A. Kent, Secure Classical Bit Commitment using Fixed Capacity Communication Channels, J. Cryptology 18 (2005) 313-335.

[10] A. Kent, A No-summoning theorem in Relativistic Quantum Theory, Quantum Information Processing 12 (2) pp 1023-1032 (2013).

[11] A. Kent, Quantum Tasks in Minkowski Space, Class. Quantum Grav. 29 (2012) 224013.

[12] P. Hayden and A. May, Summoning Information in Spacetime, or Where and When Can a Qubit Be? arXiv:1210.0913

[13] A. Kent, Unconditionally Secure Bit Commitment with Flying Qudits, New J. Phys. 13113015 (2011).

[14] A. Kent, Location-Oblivious Data Transfer with Flying Entangled Qudits, Phys. Rev. A 84, 012328 (2011).

[15] R. Colbeck and A. Kent, Variable Bias Coin Tossing, Phys. Rev. A 73, 032320 (2006).

[16] A. Kent, Quantum Tagging for Tags Containing Secret Classical Data, Phys. Rev. A 84, 022335 (2011).

[17] R. Malaney, Phys. Rev. A 81, 042319 (2010).

[18] H. Buhrman et al., arXiv:1009.2490v4 (2011).

[19] A. Kent, W. Munro and T. Spiller, Quantum Tagging: Authenticating Location via Quantum Information and Relativistic Signalling Constraints, Phys. Rev. A 84, 012326 (2011).

[20] S. Croke and A. Kent, Phys. Rev. A 86, 052309 (2012).

[21] J. Kaniewski et al., IEEE Trans. on Inf. Theory 59, 4687-4699 (2013).

[22] T. Lunghi et al., Phys. Rev. Lett. 111, 180504 (2013).

[23] Y. Liu et al., Phys. Rev. Lett. 112, 010504 (2014).

[24] T. Lunghi et al., arXiv:1411.4917.

[25] E. Adlam and A. Kent, Device-Independent Relativistic Quantum Bit Commitment, arxiv:15mm.nnnnn.

[26] A. Kent, Impossibility of unconditionally secure commitment of a certified classical bit, Phys. Rev. A 61 042301 (2000).

[27] A. Kent, Why Classical Certification is Impossible in a Quantum World, Quantum Information Processing, 11 (2), $493-499$ (2012).

[28] A. Kent, Unconditionally Secure Bit Commitment by Transmitting Measurement Outcomes, Phys. Rev. Lett. 109, 130501 (2012). 